\title{
A nurse-led intervention to improve management of virological failure in public sector HIV clinics in Durban, South Africa: A pre- and post-implementation evaluation
}

\author{
H Sunpath, ${ }^{1,2}$ MBBS, MFamMed, Dip HIV Man (SA), MPH; S Pillay, ${ }^{2}$ MSc; T J Hatlen, ${ }^{3}$ MD; R A Murphy, ${ }^{4}$ MD, MPH; \\ V C Marconi, ${ }^{5,6,7}$ MD; M-Y S Moosa, ${ }^{2}$ MB ChB, FCP, PhD; K Naidoo, ${ }^{1,6,8}$ MB ChB, Dip HIV Man (SA), PhD; M J Siedner, ${ }^{2,9,10,11}$ MD, MPH \\ ${ }^{1}$ Centre for the AIDS Programme of Research in South Africa (CAPRISA), University of KwaZulu-Natal, Durban, South Africa \\ ${ }^{2}$ Department of Infectious Diseases, Nelson R Mandela School of Medicine, College of Health Sciences, University of KwaZulu-Natal, Durban, \\ South Africa \\ ${ }^{3}$ Division of HIV, Lundquist Institute for Biomedical Innovation at Harbor-UCLA Medical Center, Torrance, Calif., USA \\ ${ }^{4}$ Division of Infectious Diseases, Lundquist Institute for Biomedical Innovation at Harbor-UCLA Medical Center, Torrance, Calif., USA \\ ${ }^{5}$ Emory University School of Medicine and Rollins School of Public Health, Atlanta, Ga., USA \\ ${ }^{6}$ Emory University Vaccine Center, Atlanta, Ga., USA \\ ${ }^{7}$ Atlanta Veterans Affairs Medical Center, Decatur, Ga., USA \\ ${ }^{8}$ Medical Research Council-Centre for the AIDS Programme of Research in South Africa (CAPRISA) HIV-TB Pathogenesis and Treatment \\ Research Unit, Doris Duke Medical Research Institute, University of KwaZulu-Natal, Durban, South Africa \\ ${ }^{9}$ Africa Health Research Institute, KwaZulu-Natal, South Africa \\ ${ }^{10}$ Medical Practice Evaluation Center, Massachusetts General Hospital, Boston, Mass., USA \\ ${ }^{11}$ Harvard Medical School, Boston, Mass., USA
}

Corresponding author: H Sunpath (henrysunpath@yebo.co.za)

\begin{abstract}
Background. Identification of patients on antiretroviral therapy (ART) with virological failure (VF) and the response in the public health sector remain significant challenges. We previously reported improvement in routine viral load (VL) monitoring after ART commencement through a health system-strengthening, nurse-led 'VL champion' programme as part of a multidisciplinary team in three public sector clinics in Durban, South Africa.

Objectives. To report on the impact of the VL champion model adapted to identify, support and co-ordinate the management of individuals with VF on first-line ART in a setting with limited electronic-based record capacity.

Methods. We evaluated the VL champion model using a controlled before-after study design. A paper-based tool, the 'high VL register', was piloted under the supervision of the VL champion to improve data management, monitoring of counselling support, and enacting of clinical decisions. We abstracted chart and electronic data (TIER.net) for eligible individuals with VF in the year before and after implementation of the programme, and compared outcomes for individuals during these periods. Our primary outcome was successful completion of the VF pathway, defined as a repeat $\mathrm{VL}<1000$ copies/mL or a change to second-line ART within 6 months of VF. In a secondary analysis, we assessed the completion of each step in the pathway.

Results. We identified 60 and 56 individuals in the pre-intervention and post-intervention periods, respectively, with VF who met the inclusion criteria. Sociodemographic and clinical characteristics were similar between the periods. Repeat VL testing was completed in $61.7 \%$ and $57.8 \%$ of individuals in these two groups, respectively. We found no difference in the proportion achieving our primary outcome in the pre- and post-intervention periods: $11 / 60$ (18.3\%; $95 \%$ confidence interval (CI) 9 - 28) and 15/56 (22.8\%; $95 \%$ CI 15 - 38), respectively $(p=0.28)$. In multivariable logistic regression models adjusted for potential confounding factors, individuals in the post-intervention period had a non-significant doubling of the odds of achieving the primary outcome (adjusted odds ratio 2.07; 95\% CI 0.75 - 5.72). However, there was no difference in the rates of completion of each step along the first-line VF cascade of care.

Conclusions. This enhanced intervention to improve VF in the public sector using a paper-based data management system failed to achieve significant improvements in first-line VF management over the standard of care. In addition to interventions that better address patient-centred factors that contribute to VF, we believe that there are substantial limitations to and staffing requirements involved in the ongoing utilisation of a paper-based tool. A prioritisation is needed to further expand and upgrade the electronic medical record system with capabilities for prompting staff regarding patients with missed visits and critical laboratory results demonstrating VF.
\end{abstract}

S Afr Med J 2021;111(4):299-303. https://doi.org/10.7196/SAMJ.2021.v111i4.15432

The identification and management of virological failure (VF) in people living with HIV (PLWH) is a cornerstone of successful HIV care. Fortunately viral load (VL) monitoring of individuals on therapy, recommended by the World Health Organization, ${ }^{[1]}$ has expanded vastly in the sub-Saharan African region. ${ }^{[2]}$ Despite this expansion, there remain significant weaknesses in identification 
of patients experiencing VF and delays in appropriate switching to second-line ART. ${ }^{[3]}$

Prompt response to individuals failing therapy decreases the risk of mortality, opportunistic infections, drug resistance and HIV transmission. ${ }^{[4-6]}$ Furthermore, management of VF is complex, particularly for over-burdened public sector clinics. Most guidelines recommend that individuals with VF undergo a complex series of clinical encounters. These include multiple visits for adherence support interventions, repeat VL testing, and visits to consider regimen changes to second-line antiretroviral therapy (ART). Notably, regimen changes to second-line ART often require approval from a senior clinical staff member. Moreover, individuals with VF represent an inherently complex patient population and often experience additional barriers to care. ${ }^{[7]}$ These complexities lead to low resuppression rates and poor outcomes in patients with $\mathrm{VF}^{[8-13]}$

Consequently, interventions to improve monitoring and management of individuals with VF are needed. We previously reported on the design of a health system-strengthening, nurse-led 'viral load champion' programme as part of a multidisciplinary team in three public sector clinics in Durban, South Africa (SA). The programme achieved a significant improvement in VL testing rates after ART initiation, ${ }^{[14]}$ but did not assess the management of patients after VF. Beginning in 2017, we piloted a paper-based tool, the 'high VL register', as part of the VL champion model to strengthen the response to VF management. We now report results of a controlled before-after study design of the pilot programme to assess whether it improved processes and clinical outcomes for PLWH with VF.

\section{Methods \\ Study setting}

The VL champion pilot programme was implemented at three public sector HIV clinics in Durban: Clairwood Hospital, Wentworth Hospital and King Dinizulu Hospital. Prior to the intervention in 2017, monitoring of patients with VF was expected to be conducted in accordance with SA National Department of Health guidelines. ${ }^{[7]}$ However, no specific clinic staff were delegated the tasks of identifying or monitoring patients with VF, which generally occurred on an ad hoc basis. A paper-based tool, the high VL register (available as a supplementary file at http://samj.org.za/public/sup/15432.pdf), was used in the VL champion model as part of a multidisciplinary team within a standard operating procedure (SOP) to monitor patient visits and assist in management of the VF cascade of care.

\section{Intervention description}

In January 2017, we implemented a health system-strengthening programme to manage VF at each of the three clinics. The programme included the following elements:

- Assignment of a nurse as the 'VL champion' at each clinic to supervise the staff responsible for monitoring all patients with a detectable VL.

- Development of an SOP for management of VF by clinic staff. Training on the SOP was provided to: (i) a lay counsellor or nurse assigned to adherence counselling; (ii) a nurse and/or doctor assigned to manage the VF clinic; (iii) an administrative clerk for records handling; and (iv) a data clerk to ensure same-day data entry. In brief, VL results were reviewed daily by the VL champion and filed or entered in the patient charts. Patient charts with a high VL result were identified with a sticker and filed separately from the remaining clinic charts by the administrative clerk. Upon return to the clinic, those marked with a high VL sticker were identified by the administrative clerk and referred directly to the counsellor, who performed adherence counselling and then referred the patient to the high VL physician or nurse. The lay counsellor also managed completion of the high VL register (http://samj.org.za/public/sup/15432.pdf) and was expected to call patients who missed a clinic appointment within a week at any point of the follow-up period.

\section{Study population and data sources}

All adult patients on first-line ART (zidovudine or tenofovir, lamivudine and efavirenz) with a VL $>1000$ copies $/ \mathrm{mL}$ were screened for eligibility from the following data sources: TIER.net, ${ }^{[15]}$ patient clinical charts, or the weekly clinic electronic dashboard of the National Health Laboratory Service. We excluded pregnant patients and those actively participating in other research studies in the clinic. Data were abstracted at the three intervention clinics during December 2015 December 2017. For each participant who met the inclusion criteria, sociodemographic data, laboratory data (CD4 count and HIV-1 RNA $\mathrm{VL}$ ), dates of clinic visits and enhanced adherence counselling (EAC) events, and dates of regimen changes were obtained.

\section{Study design and statistical methods}

We evaluated the VL champion intervention using a controlled before-after observational design. The primary exposure of interest was the outcomes in the cascade in the two periods, pre-intervention (December 2015 - December 2016) and post-intervention with the VL champion model (January 2017 - December 2017). Individuals were allocated by their first clinic visit after a VL $>1000$ copies $/ \mathrm{mL}$ during 2016 as being in the pre-intervention period, and those with their first visit after a VL $>1000$ copies/mL during 2017 as being in the post-intervention period. Those with a VL $>1000$ copies $/ \mathrm{mL}$ and no additional visits were included in the analysis and categorised by the year of their high VL.

The primary outcome was appropriate response completed to the repeat VL, specifically a $\mathrm{VL}<1000$ copies/mL or change to a protease inhibitor-based regimen after a repeat VL $>1000$ copies $/ \mathrm{mL}$ within 6 months of VF. Secondary outcomes included completion of at least one EAC session and completion of a repeat VL within 6 months.

Fisher's exact tests and $\chi^{2}$ tests were used to compare descriptive indices of individuals in the pre- and post-intervention periods. Crude rates of outcomes by intervention period were estimated and graphically depicted the 'second-line cascade of care' to describe the proportion of people with first-line VF who successfully completed $\geq 1 \mathrm{EAC}$ and a repeat VL, and had appropriate response completed. Finally, we fitted logistic regression models to identify correlates of our primary outcome with and without confounders, including age, sex, CD4 count, ART duration, ART regimen, and clinic of attendance. All data analyses were completed with Stata version 15.1 (StataCorp, USA).

\section{Ethical considerations}

The study protocol was approved by the KwaZulu-Natal Provincial Health Research Committee and the Biomedical Research Ethics Committee of the University of KwaZulu-Natal (ref. no. BFC 377/16).

\section{Resullts}

A total of 116 individuals were identified as eligible for the assessment on first-line ART with a VL >1 000 copies/mL. During the observation period, 60 individuals (including 3 with no additional visit) were eligible in the pre-intervention period and 56 (including 4 with no additional visit) in the post-intervention period (Table 1). Participants in the two periods were similar in terms of sociodemographic and clinical characteristics. The primary outcome, confirmation of a 
repeat VL $<1000$ copies/mL or a change to second-line ART within 6 months, was similar in the pre- and post-intervention groups (11/60 (18.3\%; 95\% confidence interval (CI) $9-28)$ and 15/56 (26.8\%; 95\% CI 15 - 38), respectively $(p=0.28)$ ). In multivariable logistic regression models adjusted for potential confounding factors, individuals in the post-intervention period had a non-significant doubling of the odds of achieving the primary outcome (adjusted odds ratio 2.07; 95\% CI 0.75 - 5.72) (Table 2).

We found similar trends across the firstline failure cascade of care (Fig. 1). In the preand post-intervention groups, 37/60 (61.7\%) and 29/56 (51.8\%) individuals, respectively, had a second VL done. Of those with a second VL result $<1000$ copies $/ \mathrm{mL}$, only $8.3 \%$ and $10.7 \%$, respectively, were managed as per the guidelines by continuation of firstline ART; $10.1 \%$ and $16.1 \%$, respectively, were changed to second-line ART with a $\mathrm{VL}>1000$ copies $/ \mathrm{mL}$ within 6 months of detection of VF.

\section{Discussion}

The VL champion model was developed to address barriers to improving routine VL testing and monitoring. We previously reported successful outcomes in improving VL completion rates utilising the VL champion model after ART initiation from $62 \%$ to $>90 \%$ at 1 year and maintaining high VL suppression rates. ${ }^{[14]}$ We now report the results of adapting the VL champion model to use a paper-based 'high VL register' to manage the small but significant numbers of individuals with VF. There was no significant difference in the primary outcome for the confirmation of a repeat VL $<1000$ copies/ $\mathrm{mL}$ or a change to second-line ART within 6 months of detection of VF in the pre- and post-intervention groups. In both groups, poor outcomes were reported at all stages of the VF cascade of care (number of EAC sessions, second VL and subsequent visit after repeat VL).

This study reinforces prior work demonstrating extremely poor outcomes after first-line ART failure in sub-Saharan Africa. ${ }^{[9]}$ Potentially more concerning than suboptimal VL monitoring is the startlingly low response to a detectable VL that we observed in this cohort in the preintervention and post-intervention periods.
Our findings are similar to a Mozambican study that also reported a poor cascade of care in health system response to VF. ${ }^{[1]}$ In that study, only $35 \%$ of individuals with detected VF had an appropriate repeat test, which showed $62 \%$ to have persistent VF. Only a third of those with persistent VF appropriately started second-line ART. An analysis from rural KwaZulu-Natal Province using electronic health records also reported very poor management of VF. ${ }^{[10]}$ Only $34 \%$ of patients had a VL documented 12 months after starting ART, and only $18 \%$ of these had the recommended repeat VL conducted. In total, and similar to our study, only $20 \%$ of individuals were confirmed to have

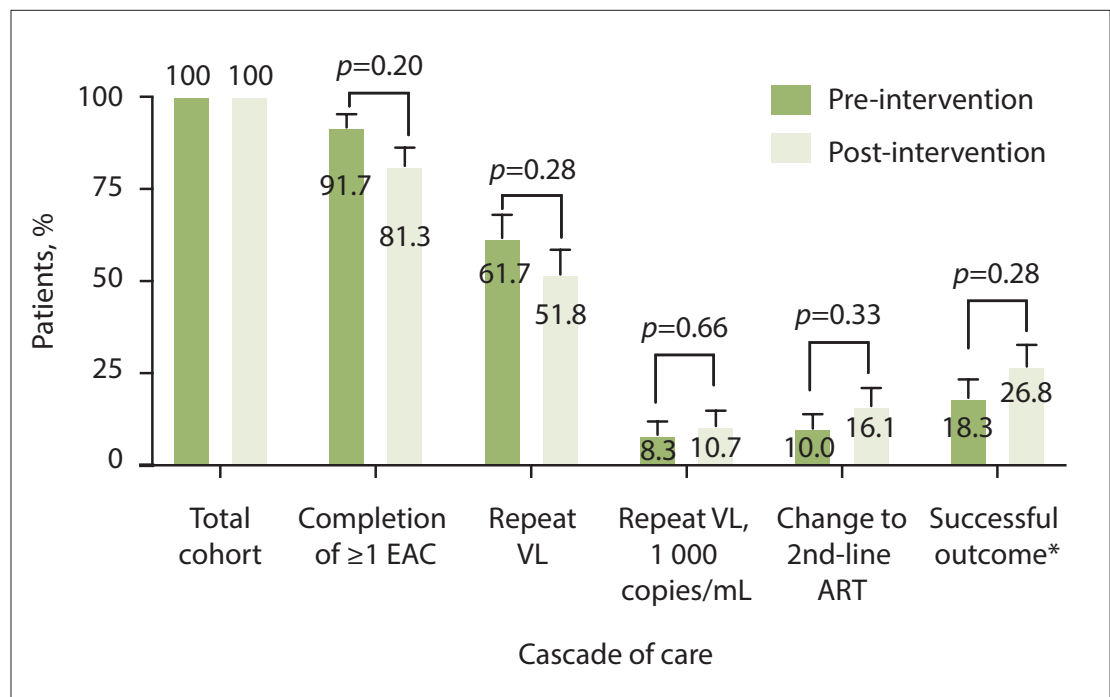

Fig. 1. Second-line cascade of care before and after implementation of a nurse-led viral load monitoring and management programme. $(E A C=$ enhanced adherence counselling; $V L=$ viral load; $A R T=$ antiretroviral therapy; ${ }^{*}$ Successful outcome defined as a repeat $V L<1000$ copies $/ m L$ or a change to second-line ART after a repeat $V L>1000$ copies $/ m L$ within 6 months of first-line ART virological failure.)

Table 1. Cohort characteristics

\begin{tabular}{llll}
\hline Characteristic & Pre-intervention period $(\boldsymbol{N}=\mathbf{6 0})$ & Post-intervention period $(\boldsymbol{N}=\mathbf{5 6})$ & $\boldsymbol{p}$-value \\
\hline Age (years), median (IQR) & $36(23-41)$ & $35(30-39)$ & 0.18 \\
Female, $n(\%)$ & $35(58.3)$ & $25(44.6)$ & 0.14 \\
ART duration (years), median (IQR) & $1.3(0.5-3.2)$ & $1.5(0.5-3.3)$ & 0.74 \\
Viral load at failure (copies/mL), median (IQR) & $19443(4751-82910)$ & $16963(5621-144977)$ & 0.64 \\
Baseline CD4 count (cells/ $\mu \mathrm{L})$, median (IQR) & $130(38-223)$ & $114(28-193)$ & 0.41 \\
IQR = interquartile range; ART $=$ antiretroviral therapy. & & &
\end{tabular}

Table 2. Logistic regression model for correlates of a successful outcome after virological failure*

\begin{tabular}{|c|c|c|c|c|}
\hline & \multicolumn{2}{|c|}{ Univariable models } & \multicolumn{2}{|c|}{ Multivariable model } \\
\hline & OR $(95 \% \mathrm{CI})$ & $p$-value & aOR $(95 \% \mathrm{CI})$ & $p$-value \\
\hline Age (each 10 years) & $0.99(0.91-1.06)$ & 0.76 & $1.35(0.78-2.35)$ & 0.29 \\
\hline Female sex & $1.81(0.86-3.81)$ & 0.12 & $3.59(1.21-10.6)$ & 0.02 \\
\hline Baseline viral load $\left(\log _{10}\right.$ copies $\left./ \mathrm{mL}\right)$ & $0.99(0.62-1.59)$ & 0.97 & $0.89(0.48-1.65)$ & 0.70 \\
\hline Pre-post intervention & $1.63(0.67-3.94)$ & 0.28 & $2.07(0.75-5.72)$ & 0.16 \\
\hline
\end{tabular}


virological resuppression or a change to second-line therapy after VF, and those who did change therapy did so a median of 1 year after VF. These results show a consistently poor response to treatment failure in the region. Additional measures are needed to better identify, monitor, and ensure more effective clinical management for this high-risk population.

There was a drop in numbers of EAC sessions and second VLs done in the post-intervention period. If adherence counselling was not performed with sufficient expertise or if staff implementation of EAC was not consistent, the remainder of the intervention could be less effective, as reported in other studies. ${ }^{[16-18]}$ The results of our intervention demonstrate that addressing health system factors alone may not be sufficient to improve VF management. We hypothesise that failure to demonstrate improved outcomes was the result of multiple factors. Attention was directed predominantly towards SOPs, training, and support for laboratory testing and results reporting. The patient-specific factors that may need to be addressed are transportation costs, work/clinical care trade-offs, stigma and mental health issues, which have all been shown to affect outcomes in this patient population. ${ }^{[19]}$ A differentiated care model, which adds focus and resources to patients with VF, might serve to improve on-treatment HIV care in the public sector. ${ }^{[20,21]}$

Both groups had similar rates of repeated VL testing, yet the number who returned for follow-up management was very low. Although the team contacted missing patients, additional measures for those who could not be found were limited by funds and staffing time. There is no inherent capacity in paper-based tools to prompt clinic staff to follow up missed visits. This system requires manual entry for reconciliation of laboratory results, visit schedules and clinical reporting. Additionally, the available electronic data repositories used in KwaZulu-Natal are not updated in real time and are often not available to nursing staff. We believe that a priority should be to upgrade the electronic medical record system with the capacity to prompt staff to track patients and universalise access to up-to-date electronic data repositories. Efforts to advance online medical record systems across SA, as successfully implemented in Western Cape Province, may meaningfully improve patient flow and clinical care. ${ }^{[22]}$

Clearly, additional novel strategies are needed to more promptly identify and switch patients who qualify for second-line ART, specifically patients with advanced HIV, the group with the worst outcomes after treatment failure. ${ }^{[23,24]}$ In addition to improving online medical record systems, a greater emphasis on task-shifting to allow nurses to change patients to second-line ART with approval from a doctor is worthy of further investigation. ${ }^{[25]}$ Another strategy currently under investigation is incorporation of resistance testing into the management of VF, which may differentiate those with failure into adherence- and resistance-based causes and could encourage clinicians to respond earlier. ${ }^{[26]}$ The roll-out of dolutegravir-based ART as first-line therapy in the region, a regimen with a higher barrier to resistance and better tolerability, is also likely to change the relative importance of the workflow components for monitoring VF. It could conceivably make poor adherence rather than ART resistance the primary determinant of treatment failure. ${ }^{[27]}$ Dolutegravir-based ART may further highlight the importance of adherence counselling in the management of VF and require further optimisation of the current VF monitoring and switch guidelines. ${ }^{[28]}$

\section{Study limitations}

Our study had limitations. The first was the small sample size, which may not have allowed sufficient power to detect a modest improvement in the outcome of interest. Second, as it was an observational study with a historical control group, there may have been unmeasured changes in patient characteristics or management that accrued over time and also had an impact on our outcomes. Third, it was an observational study that followed very soon after the establishment of a new model of care, so there may not have been sufficient time for full uptake of the new model to affect patient care outcomes. Finally, the clinics under investigation were mainly urban, and to make the findings of future studies generalisable, it will be essential to include rural clinics with different human resource profiles.

\section{Conclusions}

In summary, this study provides a basis for more comprehensive, evidence-based, clinical operational strategies that are sorely needed to improve VF management based on implementation science research. These programmes must address both health system and individual barriers to care and consider the complex mechanisms of detection, monitoring and response to VF. Until this standard of practice is available and reflected in programmatic guidelines, high rates of losses from care, delays in appropriate switching to secondline ART, and poor outcomes among people with VF will remain unacceptably common in the region, and threaten the success and durability of global HIV treatment programmes. The VL champion model improves VL completion rates after ART commencement and may be expanded as a national public health intervention, but the model for improving VF requires further implementation research.

Declaration. The research for this study was done in partial fulfilment of the requirements for HS's $\mathrm{PhD}$ degree at the University of KwaZulu-Natal. Acknowledgements. We are thankful for the substantial efforts and contributions from the patients who participated in this programme and the nurse and ancillary staff at the clinics, and our partners at the Department of Health for their support of this work.

Author contributions. HS: design of intervention, conceptualisation, manuscript preparation and finalisation. SP: data collection and management. TJH: manuscript revision. RAM: technical input into the design of the intervention, manuscript revision. VCM: design of standard operating procedure. M-YSM: supervisor of project and conceptualisation, manuscript edits. KN: supervisor of project implementation, manuscript edits. MJS: conceptualisation, data collection and analysis; manuscript drafting and revisions.

Funding. MJS receives research support from the National Institutes of Health (NIH R01 AI124718). The content of this article is solely the responsibility of the authors and does not necessarily represent the official views of the National Institutes of Health. This project has been supported by the President's Emergency Plan for AIDS Relief (PEPFAR) through the US Agency for International Development (USAID) under co-operative agreement number AID-674-A-12-00019. The contents are the sole responsibility of the authors and do not necessarily reflect the views of USAID or the US government. A PEPFAR-funded non-governmental organisation provided on-site data management support in addition to training and mentorship for EAC and project instruments.

Conflicts of interest. None.

1. World Health Organization. Guideline on when to start antiretroviral therapy and on pre-exposure prophylaxis for HIV Geneva: WHO, 2015. http://www.ncbi.nlm.nih.gov/books/NBK327115/ (accessed 5 October 2020).

2. Haas AD, Keiser O, Balestre E, et al. Monitoring and switching of first-line antiretroviral therapy in adult treatment cohorts in sub-Saharan Africa: Collaborative analysis. Lancet HIV 2015;2(7):e271-e278. https://doi.org/10.1016/S2352-3018(15)00087-9 
3. Murphy RA, Court R, Maartens G, Sunpath H. Second-line antiretroviral therapy in sub-Saharan Africa: It is time to mind the gaps. AIDS Res Hum Retroviruses 2017;33(12):1181-1184. https://doi.org/10.1089/ AID.2017.0134

4. Marconi VC, Sunpath H, Lu Z, et al. Prevalence of HIV-1 drug resistance after failure of a first highly active antiretroviral therapy regimen in KwaZulu Natal, South Africa. Clin Infect Dis 2008;46(10):15891597. https://doi.org/10.1086/587109

5. Cohen MS, Chen YQ, McCauley M, et al. Antiretroviral therapy for the prevention of HIV-1 transmission. N Engl J Med 2016;375(9):830-839. https://doi.org/10.1056/NEJMoal105243

6. TenoRes Study Group. Global epidemiology of drug resistance after failure of WHO recommended irst-line regimens for adult HIV-1 infection: A multicentre retrospective cohort study. Lancet Infect Dis 2016;16(5):565-575. https://doi.org/10.1016/S1473-3099(15)00536-8

7. Meintjes G, Moorhouse MA, Carmona S, et al. Adult antiretroviral therapy guidelines 2017. South Afr J HIV Med 2017;18(1):776. https://doi.org/10.4102/sajhivmed.v18i1.776

8. Johnston V, Fielding KL, Charalambous S, Churchyard G, Phillips A, Grant AD. Outcomes following virological failure and predictors of switching to second-line antiretroviral therapy in a South African treatment program. J Acquir Immune Defic Syndr 2012;61(3):370-380. https://doi.org/10.1097/ QAL.0b013e318266ee3f

9. Sunpath H, Naidu KK, Pillay S, Siedner MJ. The second cascade: Management of patients with high viral loads in public HIV clinics in Durban, South Africa. Poster session, 22nd International AIDS Conference, Amsterdam, 23 - 27 July 2018

10. Iwuji CC, Shahmanesh M, Koole O, et al. Clinical outcomes after first-line HIV treatment failure in South Africa: The next cascade of care. HIV Med 2020;21(7):457-462. https://doi.org/10.1111/hiv.12877

11. Swannet S, Decroo T, de Castro SMTL, et al. Journey towards universal viral load monitoring in Maputo, Mozambique: Many gaps, but encouraging signs. Int Health 2017;9(4):206-214. https://doi.org/10.1093/ inthealth/ihx021

12. Mgosha PC. Barriers to switching patients to second-line antiretroviral treatment among clinicians in Tanzania. Doctoral dissertation. Minneapolis, USA: Walden University, 2017.

13. Ssempijia V, Nakigozi G, Chang L, et al. Rates of switching to second-line antiretroviral therapy and impact of delayed switching on immunologic, virologic, and mortality outcomes among HIV-infected adults with virologic failure in Rakai, Uganda. BMC Infect Dis 2017;17(1):582. https://doi.org/10.1186/ s12879-017-2680-6

14. Sunpath H, Hatlen TJ, Naidu KK, et al. Targeting the third ' 90 ': Introducing the viral load champion. Public Health Action 2018;8(4):225-231. https://doi.org/10.5588/pha.18.0063

15. Osler M, Boulle A. Three interlinked electronic registers (TIER.Net) Project: A working Paper. September 2010. https://docplayernet/2596505-Three-interlinked-electronic-registers-tier-net-project-a-workingpaper-september-2010.html (accessed 9 March 2021).

16. Bango F, Ashmore J, Wilkinson L, van Cutsem G, Cleary S. Adherence clubs for long-term provision of antiretroviral therapy: Cost-effectiveness and access analysis from Khayelitsha, South Africa. Trop Med Int Health 2016;21(9):1115-1123. https://doi.org/10.1111/tmi.12736

17. Mukherjee JS, Barry D, Weatherford RD, Desai IK, Farmer PE. Community-based ART programs Sustaining adherence and follow-up. Curr HIV/AIDS Rep 2016;13(6):359-366. https://doi.org/10.1007/ s11904-016-0335-7
18. Fox MP, Pascoe S, Huber AN, et al. Adherence clubs and decentralized medication delivery to support patient retention and sustained viral suppression in care: Results from a cluster-randomized evaluation patient retention and sustained viral suppression in care: Results from a cluster-randomized evaluation
of differentiated ART delivery models in South Africa. PLoS Med 2019;16(7):e1002874. https://doi. of differentiated ART delivery models
org/10.1371/journal.pmed.1002874

19. Fox MP, Pascoe SIS, Huber AN, et al. Effectiveness of interventions for unstable patients on antiretroviral therapy in South Africa: Results of a cluster-randomized evaluation. Trop Med Int Health 2018;23(12):1314-1325. https://doi.org/10.1111/tmi.13152

20. Grimsrud A, Bygrave H, Doherty M, et al. Reimagining HIV service delivery: The role of differentiated care from prevention to suppression. J Int AIDS Soc 2016;19(1):21484. https://doi.org/10.7448/ IAS. 19.1.21484

21. Phillips A, Shrouf A, Vojnov L, Cohn J, Roberts T, et al. Sustainable HIV treatment in Africa through viral-load-informed differentiated care. Nature 2015;528(7580):S68-S76. https://doi.org/10.1038 nature 16046

22. Egger M. Electronic medical record systems, data quality and loss to follow-up: Survey of antiretroviral therapy programmes in resource-limited settings. Bull World Health Organ 2008;86(12):939-947. https://doi.org/10.2471/BLT.07.049908

23. Villa G, Abdullahi A, Owusu D, et al. Determining virological suppression and resuppression by point-of-care viral load testing in a HIV care setting in sub-Saharan Africa. EClinicalMedicine by point-of-care viral load testing in a HIV care setting in

24. Shroufi A, van Cutsem G, Cambiano V, et al. Simplifying switch to second-line antiretroviral therapy in sub Saharan Africa: Predicted effect of using a single viral load to define efavirenz-based first-line failure. AIDS 2019;33(10):1635-1644. https://doi.org/10.1097/QAD.0000000000002234

25. Crowley T, Mavers P. Trends in task shifting in HIV treatment in Africa: Effectiveness, challenges and acceptability to the health professions. Afr J Prim Health Care Fam Med 2015;7(1). https://dol org/10.4102/phcfm.v7i1.807

26. Siedner MJ, Bwana MB, Moosa M-YS, et al. The REVAMP trial to evaluate HIV resistance testing in sub-Saharan Africa: A case study in clinical trial design in resource limited settings to optimize effectiveness and cost effectiveness estimates. HIV Clin Trials 2017;18(4):149-155. https://doi.org/10 1080/15284336.2017.1349028

27. Cruciani M, Parisi SG. Dolutegravir based antiretroviral therapy compared to other combined antiretroviral regimens for the treatment of HIV-infected naive patients: A systematic review and meta-analysis. PLoS ONE 2019;14(9):e0222229. https://doi.org/10.1371/journal.pone.0222229

28. National Department of Health, South Africa. 2019 ART clinical guidelines for the management of HIV in adults, pregnancy, adolescents, children, infants and neonates. 2019. http://bit.ly/2019-ARTGuidelines (accessed 9 March 2021).

Accepted 6 January 2021 